\title{
AGARRE BIMANUAL DE OBJETOS ASISTIDO POR VISION
}

\author{
J.A. Castro-Vargas ${ }^{1}$, B.S. Zapata-Impata ${ }^{1,2}$, P. Gil ${ }^{1,2}$, J. Pomares $^{3}$ \\ ${ }^{1}$ Instituto Universitario de Investigación Informática, Universidad de Alicante, 03690, Alicante \\ ${ }^{2}$ Grupo AUROVA. Dep. Física, Ingeniería de Sistemas y Teoría de la Señal, Universidad de Alicante, 03690, Alicante \\ ${ }^{3}$ Grupo HURO. Dep. Física, Ingeniería de Sistemas y Teoría de la Señal, Universidad de Alicante, 03690, Alicante \\ \{jacastro,brayan.impata,pablo.gil, jpomares\}@ua.es
}

\section{Resumen}

Las tareas de manipulación de objetos, en ocasiones, requieren hacer uso de dos o más robots cooperando. En la industria 4.0 cada vez es más demandada la robótica asistencial por ejemplo para llevar a cabo tareas como levantar, arrastrar o empujar un bulto/paquete de elevado peso y/o de ciertas dimensiones. Por lo que cada vez es más frecuente encontrar robots con forma humana destinados a ayudar al operario humano en actividades en las que se dan este tipo de movimientos. En este artículo se presenta una plataforma robótica asistida por visión para llevar a cabo tareas de agarre y manipulación bimanual de objetos. La plataforma robótica consta de un torso metálico con articulación rotacional en la cadera y de dos manipuladores industriales, de 7 grados de libertad, que actúan como brazos y que a su vez montan en sus extremos sendas manos robóticas multidedo. Cada una de las extremidades superiores está dotada de percepción visual, ya que hacen uso de 2 sensores $R G B D$ ubicados en configuración eye-to-hand. La plataforma ha sido empleada y testeada con éxito para llevar a cabo agarres bimanuales de objetos en aras de poder desarrollar tareas de manipulación cooperativa de manera coordinada entre ambas extremidades.

Palabras clave: Detección de puntos de agarre, agarre bimanual, agarre cooperativo, RGBD, nubes de puntos, manipulación robótica.

\section{INTRODUCCIÓN}

La industria 4.0 demanda la realización de actividades productivas cada vez más complejas que requieren, en ocasiones, la realización de tareas de manera colaborativa entre robots y operadores humanos [2]. Además, en ocasiones el robot que colabora con el humano tiene que haber sido diseñado para llevar a cabo tareas con un alto grado de adaptabilidad. Este tipo de robots permite al humano una mejora de las condiciones laborales, evitando al operador humano posturas no ergonómicas cuando levanta objetos de cierto volumen, aunque tenga un peso contenido, o re- duciendo lesiones cuando se trata de mover objetos pesados y que requieren cierto esfuerzo físico.

En el ámbito científico e industrial se trabaja aportando soluciones basadas en sistemas multirobot individuales, o en dos o más sistemas multirobot capaces de funcionar de manera individual, pero también de hacerlo de manera conjunta para desarrollar una tarea de modo cooperativo, donde tienen cabida los manipuladores industriales bimanuales [13]. En el pasado, la robótica industrial ya resolvió el problema de mover objetos pesados y voluminosos, construyendo grandes robots industriales, capaces de manipular un objeto pero donde la colaboración junto al humano se hacía imposible por peligrosidad y espacio de trabajo requerido por el robot.

En los últimos años se están introduciendo en los entornos de trabajo robots colaborativos mucho más pequeños y en una gran mayoría de casos con apariencia similar al humano pero dotados de un único brazo robótico como por ejemplo TIAGo de PAL robotics [9] y SAWYER de Rethink robotics [8] o dotados con dos brazos robóticos como TALOS [14] y BAXTER [10] de los dos mismos fabricantes. Estos últimos al estar dotados de dos extremidades permiten llevar a cabo tareas cooperativas entre ambos brazos. No obstante, el diseño y materiales de construcción limitan la aplicabilidad cuando el objeto no es ligero o de pequeñas dimensiones. En estos casos, hay soluciones más industriales como el robot YUMI-IRB 14000 de ABB (hasta 500gr) o el robot LBR-iiwa de KUKA (hasta $14 \mathrm{~kg}$ en el modelo R820) como es discutido en $[6]$.

La manipulación bimanual es una tarea que abarca diferentes etapas, como son la planificación del agarre, el control del robot y la percepción del agarre, entre otros. El trabajo aquí presentado se centra principalmente en la primera etapa, y más concretamente en estimar puntos de contacto para garantizar un agarre inicial bimanual estable [7], que permita levantar un objeto sin llevar a cabo una adaptación del agarre durante la manipulación dextrogira de un objeto.

En la industria 4.0, los robots aún se enfrentan 
con dificultades cuando se trata de agarrar objetos con formas, tamaños y materiales distintos. Para conseguir robots autónomos capaces de realizar agarres en estos entornos cambiantes, se hace imprescindible el uso de información extraída de sensores integrados en el robot [1] siendo frecuente los sensores de fuerza [12], táctiles [5], sistemas de visión [3], así como una combinación de algunos o de todos ellos. En esta línea, nuestro trabajo presenta una aproximación para llevar a cabo un agarre inicial bimanual que está basada, únicamente, en datos procedentes de un sistema de visión.

El presente trabajo se estructura de la siguiente manera. En la siguiente sección se contextualiza la técnica aplicada. La sección 3 describe la arquitectura robótica del sistema empleada en la experimentación, que incluye tanto los robots como el sistema de percepción visual. En la sección 4, se describe el método de detección de puntos de agarre para tareas de agarre bimanual. En la sección 5 se presenta la planificación del agarre bimanual y posteriormente, los experimentos llevados a cabo para mostrar el funcionamiento del sistema. Finalmente en la sección 6 se muestran las conclusiones.

\section{TRABAJOS RELACIONADOS}

En el estado del arte es posible encontrar tres tipos de aproximaciones basadas en visión para calcular puntos de agarre sobre un objeto.

1. Reconstrucción de objetos: Algunos autores han propuesto bases de datos con agarres precalculados sobre figuras geométricas básicas [16]. Durante un agarre real en el sistema robótico, el robot descompone una imagen RGBD para obtener reconstrucciones de formas geométricas como cilindros o cubos. Entonces, estas partes del objeto real se utilizan para buscar correspondencias con las geometrías básicas. Una vez se encuentra una semejanza, se utilizan las estrategias predefinidas sobre como agarrar dicha forma geométrica.

2. Aprendizaje automático: La estimación de puntos de agarre se transforman en un problema de clasificación. Así, en [4] se introduce la idea de lo que se denomina el rectángulo de agarre: un rectángulo con una orientación en el espacio de una imagen 2D, donde dos de sus lados representan las pinzas robóticas y los otros dos representan la apertura de dicha pinza. Con ello, la tarea se transforma en clasificar estos rectángulos según la calidad del agarre que resultaría de ubicar la pinza robótica en la posición del rectángulo sobre el objeto.

3. Análisis de nubes de puntos: Se emplean nubes de puntos 3D obtenidas, habitualmente, a partir de sensores RGBD o tiempo de vuelo (ToF: Time of Flight) porque éstas tienen la ventaja de contener mucha más información sobre el volumen, la superficie y la localización de los objetos que la simple composición RGBD o de imágenes 2D. En [11] se lleva a cabo uno de los primeros trabajos donde se propone calcular los puntos de contacto usando este tipo de datos. Sus autores proponen buscar primero la superficie plana más alta del objeto y entonces calculan el punto más cercano al centroide en el borde del objeto. El segundo punto de agarre lo definen en el lado opuesto. Recientemente, en [15] se proponen buscar configuraciones antipodales de la pinza robótica sobre el la nube de puntos 3D del objeto. De esta manera, consiguen puntos de contacto donde la pinza puede realizar agarres estables. Estas configuraciones son luego clasificadas por una red convolucional (CNN) que es la encargada de predecir las posibilidades de éxito en el agarre. Hay que hacer notar que en todos estos trabajos, la experimentación nunca es bimanual.

En nuestro trabajo se sigue la tercera metodología presentada y es aplicada a agarre bimanual. Nosotros utilizamos dos sensores visuales con los que registramos dos nubes de puntos $3 \mathrm{D}$, cada uno de ellos facilita una vista parcial de los objetos presentes en el área de trabajo. Además, conviene mencionar que nuestro método trabaja con objetos desconocidos. Esto es, el sistema no ha sido entrenado para agarrar objetos a partir de objetos conocidos, ni se utilizan modelos 3D de los objetos a agarrar para realizar los cálculos de los puntos de agarre.

\section{ARQUITECTURA DEL SISTEMA}

En el desarrollo de este trabajo han sido utilizados 2 robots manipuladores PA10 de siete grados de libertad (Tabla 1), montados ambos sobre un estructura sólida articulada que hace de torso (Tabla 2). La estructura del torso dispone de un grado de libertad para dotar al sistema robótico de movimientos rotacionales de cadera. Cada brazo monta en su extremo una mano Allegro, zurda y diestra respectivamente, para realizar los agarres. Ambas manos están dotadas de cuatro dedos articulados con cuatro grados de libertad cada una. En este trabajo, sólo se emplean tres de los dedos 
para garantizar un agarre estable y a la vez evitar la complejidad de planificar los algoritmos de manipulación con 4 dedos. En particular, se emplea el pulgar, el indice y el corazón, prescindiendo del anular. La Figura 1 muestra el sistema robótico bimanual formado por los $2 P A-10$, las 2 manos Allegro y el torso articulado.

Tabla 1: Denavit-Hartenberg de cada PA10.

\begin{tabular}{|c|c|c|c|c|}
\hline Articulación & $\mathbf{a}_{\mathbf{i}}$ & $\mathbf{d}_{\mathbf{i}}$ & $\alpha_{\mathbf{i}}$ & $\theta_{i}$ \\
\hline GS1/WS1 & 0 & $315 / 317$ & $-\pi / 2$ & $\theta_{1}$ \\
\hline GS2/WS2 & 0 & 0 & $\pi / 2$ & $\theta_{2}$ \\
\hline GS3/WS3 & 0 & $450 / 450$ & $-\pi / 2$ & $\theta_{3}$ \\
\hline GE1/WE1 & 0 & 0 & $\pi / 2$ & $\theta_{4}$ \\
\hline GE2/WE2 & 0 & $500 / 480$ & $-\pi / 2$ & $\theta_{5}$ \\
\hline GW1/WW1 & 0 & 0 & $\pi / 2$ & $\theta_{6}$ \\
\hline GW2/WW2 & 0 & $80 / 70$ & $-\pi / 2$ & $\theta_{7}$ \\
\hline
\end{tabular}

Tabla 2: Denavit-Hartenberg del torso.

\begin{tabular}{|l|c|c|c|c|}
\hline & $\mathbf{a}_{\mathbf{i}}$ & $\mathbf{d}_{\mathbf{i}}$ & $\alpha_{\mathbf{i}}$ & $\theta_{i}$ \\
\hline Tronco (T) & 0 & $d_{1}$ & $-\pi / 2$ & $\theta_{1}$ \\
\hline PA10 Izquierdo & $a_{2}$ & $d_{2}$ & $-\pi / 3$ & $-\pi / 2$ \\
\hline PA10 Derecho & $a_{3}$ & $d_{3}$ & $\pi / 3$ & $\pi / 2$ \\
\hline
\end{tabular}

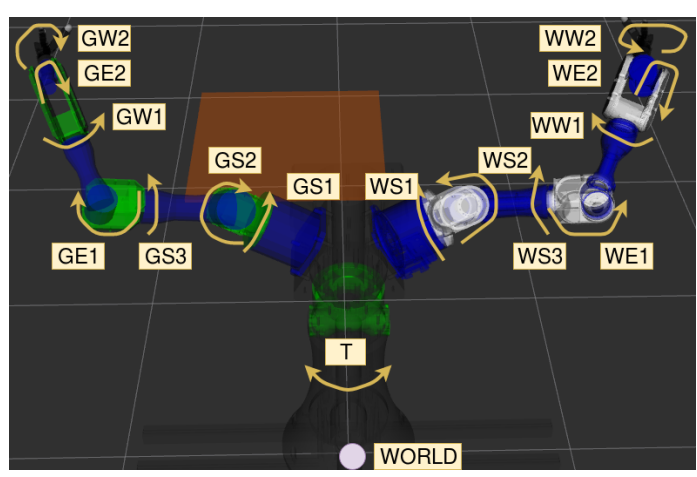

Figura 1: Robot bimanual

Además, en cada lateral del sistema robótico se dispone de una cámara RGBD con configuración eye-to-hand para recibir información de la escena. Concretamente, se han utilizado dos Intel realsense $S R 300$ con un rango trabajo de 0.2 a 1.5 metros, diseñadas para trabajar en interiores. Las cámaras son usadas para detectar las nubes de puntos de objetos en la escena primero y posteriormente estimar los puntos de agarre sobre las nubes de puntos de cada uno de los objetos.

\subsection{SISTEMA ROBOTICO BIMANUAL}

Para el control del sistema robótico, anteriormente comentado, se utiliza la plataforma software ROS (Robot Operating System) y en parti- cular, el paquete integrado MoveIt. ROS ha facilitado la integración del algoritmo propuesto en una misma unidad de control permitiendo tanto la comunicación con los dos brazos como con las dos manos robóticas, gestionando el conjunto brazo y mano robótica como una única cadena cinématica cuando se requiere, y funcionando cada conjunto como una extremidad de tren superior tratando de emular el comportamiento humano. Además, ROS habilita a nuestro algoritmo para planificar trayectorias ambas extremidades superiores en base a la información visual proporcionada por las dos cámaras RGBD.

A partir de la información de cada una de las cámaras RGBD, se obtiene puntos de agarre sobre el objeto para cada una de las manos robóticas. $\mathrm{Y}$ a partir de las posiciones $\mathbf{p}_{\mathbf{o c}}=\left(\mathbf{x}_{\mathbf{o c}}, \mathbf{y}_{\mathbf{o c}}, \mathbf{z}_{\mathbf{o c}}\right)$ de cada uno de los puntos de agarre estimados, con respecto a las cámaras RGBD, se determina la posición y orientación que deben alcanzar los dedos de la mano mediante el producto del vector de las posiciones por la matriz de transformación de coordenadas de cámara a robot $\mathbf{T}_{\mathbf{r}}^{\mathbf{c}}$. Después, haciendo uso de Moveit se planifican y evalúan trayectorias para alcanzar cada punto $\mathbf{p}_{\mathbf{o r}}=\left(\mathbf{x}_{\mathbf{o r}}, \mathbf{y}_{\text {or }} \mathrm{y}, \mathbf{z}_{\mathbf{o r}}\right)$ logrando la pose correcta de la mano para ejecutar con éxito el agarre.

Sin embargo, la planificación del movimiento cambia cuando los dedos de ambas manos robóticas han alcanzado los puntos $\mathbf{p}_{\text {or }}$, y han sido adecuadamente orientadas hasta agarrar el objeto correctamente. En esta situación, si se quiere ejecutar un movimiento y aplicarlo al objeto, tal como cambiar la orientación de éste o desplazarlo hacia otra posición es necesario que ambos brazos trabajen en sintonía, como una única cadena cinématica para todo el conjunto articular. Para conseguirlo se ha definido el objeto agarrado por los brazos como un eslabón adicional de la cadena cinemática, de modo que al cambiar la posición espacial $\mathrm{u}$ orientación del objeto los brazos respondan trabajando conjuntamente como un único robot.

\subsection{SISTEMA DE PERCEPCIÓN VISUAL}

El sistema de percepción visual, en la actualidad, consta de dos cámaras RGBD situados en los laterales del robot con una configuración eye-to-hand y con un campo visual orientado hacia el área de trabajo de los robots. En el área de trabajo se dispone de una mesa, de altura regulable, sobre la que sitúan los objetos susceptibles de ser manipulados. El empleo de dos cámaras viene motivado por conseguir un punto de vista lo más adecuado posible para observación de la escena 
y obtener trayectorias adecuadas para cada extremidad robótica. Así, cada punto de vista complementa posibles pérdidas de información debido a oclusiones entre los objetos y proporciona información de las características de los objetos para realizar una agarre efectivo por cada mano. Nuestro algoritmo se ejecuta de manera independiente para cada una de las nubes de puntos adquiridas por cada una de las cámaras. De modo que se dispone de dos flujos visuales paralelos de procesamiento. El resultado del procesamiento de ambos flujos es la obtención de dos conjuntos de puntos de agarre $\mathbf{p}_{\mathbf{o c}}$ y $\mathbf{p}_{\mathbf{o c}}^{\prime}$, uno para cada cámara. No obstante, debido a las interferencias que el infrarrojo de una cámara causa sobre la otra se requiere que durante la captura de nubes de puntos se conmute entre ambas cámaras el encendido y apagado de los emisores de luz infrarroja. De modo que el tiempo de adquisición no es igual en ambas cámaras, hay un pequeño desfase, entre el frame $\mathbf{I}$ de una cámara y el mismo frame $\mathbf{I}^{\prime}$ en la otra cámara.

A continuación en la Sección 4 se expone la metodología para estimar los agarres a partir de las nubes de puntos generadas a partir de los frames de cada cámara y así, posteriormente planificar el movimiento de agarre y su ejecución (Sección 5).

\section{ESTIMACION DEL AGARRE EN NUBES DE PUNTOS}

Para realizar el cálculo de los puntos de agarre sobre los objetos se parte de las nubes de puntos de cada frame $\mathbf{I}$ y $\mathbf{I}^{\prime}$ proporcionadas por cada una de las cámaras respectivamente (Figura 2(arriba)). Primero, se segmenta cada una de las nubes de puntos para encontrar la superficie sobre la que están apoyados los objetos y eliminar otros planos como podría ser el fondo de la escena (Figura 2(abajo-izquierda)). El resultado son grupos de puntos que conforman nubes de puntos más pequeñas y no conectadas entre sí. Cada una de estas nuevas nubes $\mathbf{P}_{\mathbf{O}_{\mathbf{i}}}$ y $\mathbf{P}_{\mathbf{O}_{\mathbf{i}}}^{\prime}$ (donde $i$ indica el identificador de objeto) constituyen un objeto distinto para cada cámara. Después, y previamente a estimar los puntos de agarre para cada robot en las distintas nubes $\mathbf{P}_{\mathbf{O}_{\mathbf{i}}}$ y $\mathbf{P}_{\mathbf{O}_{\mathbf{i}}}^{\prime}$ se aplica un filtro estadístico con el fin de eliminar puntos ruidosos y suavizar la geometría de las nubes.

En segundo lugar, para cada objeto detectado determinados por $\mathbf{P}_{\mathbf{O}_{\mathbf{i}}}$ y $\mathbf{P}_{\mathrm{O}_{\mathbf{i}}}^{\prime}$, se calcula el eje principal eoi y $\mathbf{e}_{\mathbf{O} \mathbf{i}}^{\prime}$, que proporciona una estimación de la orientación de cada objeto que representa (está de pié, acostado por una cara, de pie e inclinado, etc.). Para ello, se aplica la técnica de Análisis de Componentes Principales ( $P C A$, de sus siglas en inglés) para encontrar ese eje mayor en los puntos del objeto. Posteriormente, se calcula el centroide de cada nube de objeto, $\mathbf{c}_{\mathbf{O} \text { i }}$ y $\mathbf{c}_{\text {Oi }}^{\prime}$, que será de utilidad para tener una estimación del centro de masas del objeto.

La idea principal es calcular puntos de agarre que configuren un agarre de la mano robótica de forma que la muñeca de ésta esté perpendicular al objeto y cerca de su centro de masas, para así conseguir realizar agarres estables. Esto es porque se asume que los objetos son sólidos y tienen una masa distribuida uniformemente. En consecuencia, el eje del objeto aproximado por PCA y el centroide, nos permiten calcular el plano de corte perpendicular al objeto que lo corta por su centroide. En nuestro caso, se considera que las áreas potenciales de agarre más estable deben estar entonces en los bordes opuestos del objeto que están cerca de este plano perpendicular (Figura 2(abajocentro)). Por lo tanto, se define como configuración de agarre a la pareja formada por un punto de cada una de estas dos áreas (Figura 2(abajoderecha)).

Finalmente, cada configuración de agarre es entonces evaluada con una métrica que mide la posibilidad de que el agarre sea estable, dado el punto de vista que tenemos del objeto con la nube de puntos y la mano robótica utilizada. Está métrica fue evaluada por los autores en [17] y se calcula considerando los siguientes premisas:

1. Distancia de cada punto de agarre con el plano de corte al eje de la altura del objeto. Es decir, el plano que intersecciona perpendicularmente sobre el eje del objeto. Cuanto más cerca estén estos puntos al plano de corte, más cerca estarán los puntos a una aproximación del centro de masas del objeto. Esto es deseable para que al ejercer fuerza con la mano robótica, esta fuerza esté cerca de dicho centro y así evitar que el objeto se balancee en la mano.

2. Curvatura en los puntos de agarre. Nuestro algoritmo mide la curvatura de los puntos en las áreas potenciales de agarre para poder evaluar si los dedos se apoyarán en una superficie plana o no. Nuestra métrica evaluará mejor aquellas configuraciones que posicionen la mano sobre áreas planas, ya que estas aportarán una mayor área de contacto entre el objeto y los dedos del robot, por lo que el agarre será más estable.

3. Configuración antipodal. Las configuraciones de agarre deben proponer puntos de contacto donde la mano robótica pueda aplicar fuerzas 
colineales y opuestas, lo cual asegura una mayor estabilidad del objeto en la mano.

4. Perpendicularidad de los puntos. Los puntos de agarre deben estar conectados por una línea que es paralela a la superficie del plano de corte calculado, para formar agarres paralelos a este plano y no en diagonal. El plano es perpendicular al eje del objeto, así que para asegurar que el agarre es perpendicular al objeto se debe disponer de puntos de agarre lo más paralelos a este plano. De otra forma, el agarre tendería a ser paralelo al eje del objeto, por lo que habría mayor riesgo de deslizamientos.

En resumen, el objetivo de esta métrica es puntuar mejor aquellas configuraciones de agarre que sitúan la mano robótica con su palma mirando hacia el objeto, con sus dedos perpendiculares al eje de dicho objeto, paralelos al plano de corte calculado y cercanos al centroide. Con estas básicas condiciones geométricas, podemos encontrar agarres estables para cualquier objeto detectado por el sistema de visión.
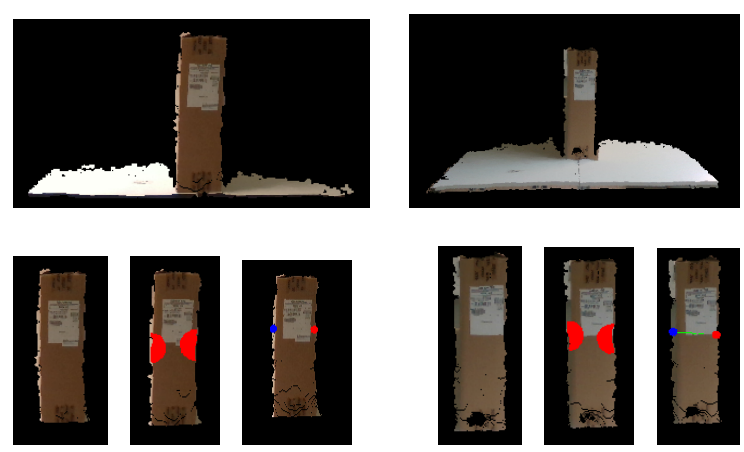

Figura 2: Escena con nubes de puntos des ambas cámaras $\mathbf{P}_{\mathbf{O}_{\mathbf{i}}}$ y $\mathbf{P}_{\mathbf{O}_{\mathbf{i}}}^{\prime}$ (arriba) y fases del algoritmo (abajo), en el que de izquierda a derecha se muestra la segmentación, detección de zona candidata de agarre en base a ejes $\mathbf{e}_{\mathbf{O}_{\mathbf{i}}}$ y $\mathbf{e}_{\mathbf{O} \mathbf{i}}^{\prime} \mathrm{y}$ centros $\mathbf{c}_{\mathbf{O}_{\mathbf{i}}}$ y $\mathbf{c}_{\mathbf{O} \mathbf{i}}^{\prime}$, así como la selección óptima de puntos de agarre $\mathbf{p}_{\mathbf{o}_{\mathbf{c}}}$ y $\mathbf{p}_{\mathbf{o c}}^{\prime}$ dentro de las zonas candidatas en base la métrica diseñada.

\section{PLANIFICACION BIMANUAL PARA EL AGARRE}

El proceso de planificación bimanual utiliza la localización de los puntos de agarre obtenidos por el sistema de percepción (Sección 4), una vez transformados al sistema del robot (Sección 3), $\mathbf{p}_{\text {or }}=\left(\mathbf{x}_{\mathbf{o r}}, \mathbf{y}_{\text {or }} \mathbf{y}, \mathbf{z}_{\text {or }}\right)$ para determinar la pose correcta para ambas manos y así llevar a cabo un agarre bimanual existoso.
El proceso de agarre bimanual consta de varias fases observables en la Figura 4. El sistema recoge información RGBD de las cámaras y las procesa para obtener puntos de agarre para cada una de las manos robóticas, de acuerdo al algoritmo presentado en la sección 4. Al mismo tiempo, se calculan las dimensiones aproximadas del objeto presente en la escena. Para ello, se calcula el bounding box 3D orientado que envuelve las nubes de puntos que representan las vistas parciales de los objetos $\mathbf{b b}\left(\mathbf{P}_{\mathbf{O}_{\mathbf{i}}}\right)$ y $\mathbf{b b}\left(\mathbf{P}_{\mathbf{O}_{\mathbf{i}}}^{\prime}\right)$. Después, se calcula la distancia euclídea entre ambos bounding box como la distancia entre los centroides $\mathbf{c}_{\mathbf{O}}$ y $\mathbf{c}_{\mathbf{O}}^{\prime}$ previamente calculados. Esa distancia determina la anchura del objeto. Posteriormente, el punto de corte de cada eje principal $\mathbf{e}_{\mathbf{O}_{\mathbf{i}}}$ y $\mathbf{e}_{\mathbf{O i}}^{\prime}$ con los correspondientes $\mathbf{b b}\left(\mathbf{P}_{\mathbf{O}_{\mathbf{i}}}\right)$ y $\mathbf{b b}\left(\mathbf{P}_{\mathbf{O}_{\mathbf{i}}}^{\prime}\right)$ determinan la altura del objeto. De modo que es posible representar un bounding box virtual que casi envuelve a $\operatorname{los} \mathbf{b b}\left(\mathbf{P}_{\mathbf{O}_{\mathbf{i}}}\right)$ y $\mathbf{b b}\left(\mathbf{P}_{\mathbf{O}_{\mathbf{i}}}^{\prime}\right)$ de cada vista del objeto, y sus dimensiones aproximadas (altura y anchura) se asemejan bastante a la altura del objeto y la anchura de éste. Este bounding box virtual $\mathbf{b b v}_{\mathbf{O}_{\mathbf{i}}}$ es empleado para aproximar el tamaño del objeto real a partir de ambas vistas parciales.

Es importante determinar las dimensiones del objeto porque la configuración de agarre bimanual depende, tanto del tamaño del objeto como de las dimensiones de las manos robóticas que se empleen. En nuestro caso, las manos robóticas Allegro tienen unas dimensiones de $248 \mathrm{~mm}$ de longitud entre el extremo del dedo y la muñeca con la mano abierta y una anchura aproximada de $242 \mathrm{~mm}$ con la mano extendida y todos los dedos empleando la máxima separación posible. En este trabajo, los objetos que se han considerado son del tipo paquete, como los que se gestionan en un almacén. Por sus formas geométricas, los casos básicos que se pueden dar se reducen a objetos apoyados por la cara de mayor tamaño (objeto acostado) o apoyados por la cara de menor tamaño (objeto de pié). En el primer caso, $\mathbf{b b v}_{\mathbf{O}_{\mathbf{i}}}$ será un objeto ancho (más anchura que altura), mientras que en el segundo caso, $\mathbf{b b v}_{\mathbf{O}_{\mathbf{i}}}$ será un objeto angosto (más altura que anchura).

Esta tipología de objeto, determina la configuración de agarre bimanualmente robótico (Figura 4). Si el objeto es ancho (i.e. objeto acostado), las manos agarrarán posicionándose de acuerdo a los puntos calculados para cada brazo según la vista asociada $\mathbf{p}_{\text {or }} \mathrm{y} \mathbf{p}_{\text {or }}^{\prime}$. Se considera esa configuración de agarre si su anchura es inferior a la suma de las longitudes de ambas manos. Si por el contrario, el objeto es alto (i.e. objeto de pié) habrá que modificar la manera de estimar los puntos de agarre, modificando ligeramente con una etapa adicional, el algoritmo de la sección 3. La idea, en este caso, 

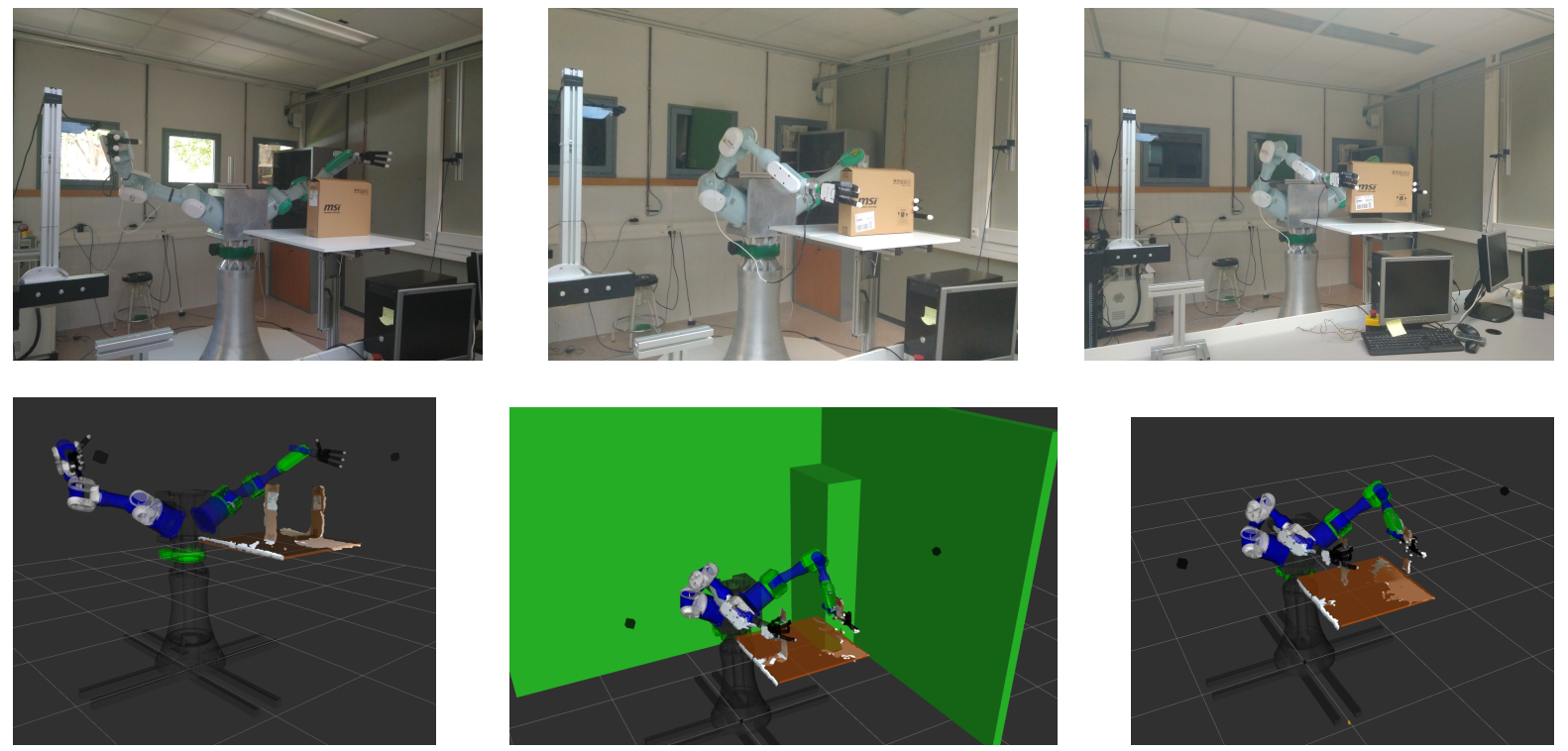

Figura 3: En las figura se observa en la parte de arriba el robot real y en la de abajo el robot virtual. De izquierda a derecha se observan las fases para ejecución de la planificación sobre un objeto ancho. Primera columna pre-agarre, segunda columna sujeción del objeto y última columna levantamiento del objeto.

consiste en particionar tanto $\mathbf{P}_{\mathbf{O}_{\mathbf{i}}}$ como $\mathbf{P}_{\mathbf{O}_{\mathbf{i}}}^{\prime}$ en dos clústeres $\mathbf{P} \mathbf{1}_{\mathbf{O}_{\mathbf{i}}}$ y $\mathbf{P} \mathbf{2}_{\mathbf{O}_{\mathbf{i}}}$ para una vista y $\mathbf{P} \mathbf{1}_{\mathbf{O}_{\mathbf{i}}}^{\prime}$ y $\mathbf{P} \mathbf{2}_{\mathbf{O}_{\mathbf{i}}}^{\prime}$ para la otra vista. La partición se lleva a cabo generando un plano que corta a $\mathbf{e}_{\mathbf{O}_{\mathbf{i}}} \mathrm{y} \mathbf{e}_{\mathbf{O} \mathbf{i}}^{\prime}$ y contiene $\mathbf{c}_{\mathbf{O}_{\mathbf{i}}}$ y $\mathbf{c}_{\mathbf{O} \mathbf{i}}^{\prime}$. Una vez particionada las nubes, para cada uno de los fragmentos, se procede de la misma manera a como se ha hecho en la sección 3, calculando el nuevo centroide y posteriormente la región candidata de agarre y los puntos que mejor ratio tienen en ella. De este modo, es posible evitar colisiones frontales entre ambas manos, garantizando que la zona de agarre de una mano esté desplazada hacia arriba o hacia abajo con respecto de la otra lo suficiente como para tratar de mantener la estabilidad en el agarre. Hay que notar que la estimación de puntos de agarre comentados en la sección 3, consideran la anchura de las manos como uno de los parámetros que se ponderan para determinar los mejores puntos de agarre. En la Figura 3 puede observarse la ejecución del sistema sobre un objeto ancho.

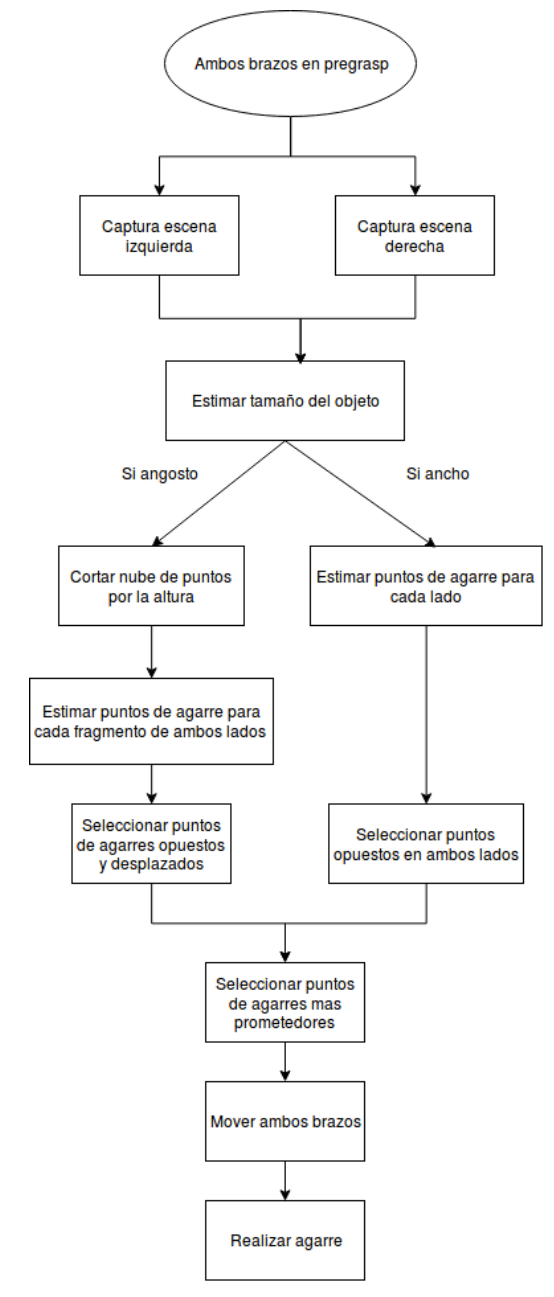

Figura 4: Diagrama explicativo de la planificación bimanual. 


\section{CONCLUSIONES}

En este trabajo se ha presentado un sistema robótico bimanual para el agarre asistido por visión. El sistema presentado usa técnicas de visión 3D para determinar puntos de agarre a partir de dos vistas parciales de la escena. El algoritmo de detección de puntos de agarre evalúa la superficie del objeto proporcionada por las dos vistas para determinar primero regiones candidatas y después potenciales puntos dentro de éstas. Las regiones se estiman considerando factores que influyen en la estabilidad del agarre. Posteriormente, el sistema robótico bimanual propuesto se ha probado en actividades de agarre de objetos del tipo paquete, como los que se manipulan en la industria logística y de almacenaje. En el futuro, se pretende analizar el comportamiento del sistema en tareas complejas de manipulación como las que se requieren en la industria 4.0 para automatizar la actividad de almacenaje de paquetes u otro tipo de objetos.

\section{Agradecimientos}

Este trabajo ha sido financiado por el proyecto europeo COMMANDIA (SOE2/P1/F0638) que está cofinanciado por el programa Interreg-V Sudoe y el Fondo Europeo de Desarrollo Regional, así como por el proyecto nacional DPI2015-68087-R.

\section{English summary}

\section{BIMANUAL GRASPING OF OB- JECTS ASSISTED BY VISION}

\author{
Abstract \\ Manipulation tasks of objects, sometimes, \\ requires the use of two or more cooperat- \\ ing robots. In the industry 4.0, assistance \\ robotic is being more and more demanded, \\ for example, to carry out tasks such as lift- \\ ing, dragging or pushing of both heavy and \\ big packages. Consequently, it is possible \\ to find robots with human appearance ad- \\ dressed on helping human operators in ac- \\ tivities in which these types of movements \\ occur. In this article, a vision-assisted \\ robotic platform is presented to carry out \\ both grasping tasks and bimanual manip- \\ ulation of objects. The robotic platform \\ consists of a metallic torso with rotational \\ joint at the hip and two industrial manipu- \\ lators, with 7 degrees of freedom, which act
}

as arms. Each arm mounts a multi-finger robotic hand at the end.Each of the upper extremities use visual perception from 3 RGBD sensors located in an eye-to-hand configuration. The platform has been successfully used and tested to carry out bimanual object grasping in order to develop cooperative manipulation tasks in a coordinated way between both robotic extremities.

Keywords: Detection of grasping points, bimanual grasping, cooperative grasping, RGBD, point clouds, robotic manipulation.

\section{Referencias}

[1] Bagnell, J. A., Cavalcanti, F., Cui, L., Galluzzo, T., Hebert, M., Kazemi, M., Klingensmith, M., Libby, J., Liu, T. Y., Pollard, N., et al. (2012). An integrated system for autonomous robotics manipulation. In Proceedings of the IEEE/RSJ International Conference on Intelligent Robots and Systems (IROS), pages 2955-2962. IEEE.

[2] Dömel, A., Kriegel, S., Kaßecker, M., Brucker, M., Bodenmüller, T., and Suppa, M. (2017). Toward fully autonomous mobile manipulation for industrial environments. International Journal of Advanced Robotic Systems, 14(4):1-19.

[3] Hudson, N., Ma, J., Hebert, P., Jain, A., Bajracharya, M., Allen, T., Sharan, R., Horowitz, M., Kuo, C., Howard, T., et al. (2014). Modelbased autonomous system for performing dexterous, human-level manipulation tasks. $A u$ tonomous Robots, 36(1-2):31-49.

[4] Jiang, Y., Moseson, S., and Saxena, A. (2011). Efficient grasping from rgbd images: Learning using a new rectangle representation. In Proceedings of the IEEE International Conference on Robotics and Automation (ICRA), pages 3304-3311.

[5] Kappassov, Z., Corrales, J.-A., and Perdereau, V. (2015). Tactile sensing in dexterous robot hands. Robotics and Autonomous Systems, 74:195-220.

[6] Khalid, A., Kirisci, P., Ghrairi, Z., Thoben, K.-D., and Pannek, J. (2016). A methodology to develop collaborative robotic cyber physical systems for production environments. Logistics Research, 9(1):23:1-15.

[7] León, B., Morales, A., and Sancho-Bru, J. (2014). Robot grasping foundations. In From 
Robot to Human Grasping Simulation, volume 19, pages 15-31. Springer.

[8] Mucchiani, C., Kennedy, M., Yim, M., and Seo, J. (2018). Object picking through in-hand manipulation using passive end-effectors with zero mobility. IEEE Robotics and Automation Letters, 3(2):1096-1103.

[9] Pages, J., Marchionni, L., and Ferro, F. (2016). Tiago: the modular robot that adapts to different research needs. In Proceedings of IEEE/RSJ International Conference on Intelligent Robots and Systems (IROS)-Workshop on Robot Modularity. IEEE.

[10] Pinto, L. and Gupta, A. (2016). Supersizing self-supervision: Learning to grasp from $50 \mathrm{k}$ tries and 700 robot hours. In Proceedings of IEEE International Conference on Robotics and Automation (ICRA), pages 3406-3413. IEEE.

[11] Richtsfeld, M. and Vincze, M. (2008). Grasping of unknown objects from a table top. In Workshop on Vision in Action: Efficient strategies for cognitive agents in complex environments.

[12] Righetti, L., Kalakrishnan, M., Pastor, P., Binney, J., Kelly, J., Voorhies, R. C., Sukhatme, G. S., and Schaal, S. (2014). An autonomous manipulation system based on force control and optimization. Autonomous Robots, 36(1-2):1130.

[13] Smith, C., Karayiannidis, Y., Nalpantidis, L., Gratal, X., Qi, P., Dimarogonas, D. V., and Kragic, D. (2012). Dual arm manipulation-a survey. Robotics and Autonomous systems, 60(10):1340-1353.

[14] Stasse, O., Flayols, T., Budhiraja, R., Giraud-Esclasse, K., Carpentier, J., Del Prete, A., Soueres, P., Mansard, N., Lamiraux, F., Laumond, J.-P., et al. (2017). Talos: A new humanoid research platform targeted for industrial applications. pages 689-695.

[15] ten Pas, A., Gualtieri, M., Saenko, K., and Platt, R. (2017). Grasp pose detection in point clouds. The International Journal of Robotics Research, 36(13-14):1455-1473.

[16] Vahrenkamp, N., Westkamp, L., Yamanobe, N., Aksoy, E. E., and Asfour, T. (2016). Partbased grasp planning for familiar objects. In Proceedings of the 16th International Conference on Humanoid Robots (Humanoids), pages 919-925. IEEE.

[17] Zapata-Impata, B. S., Mateo, C. M., Gil, P., and Pomares, J. (2017). Using geometry to detect grasping points on $3 \mathrm{~d}$ unknown point cloud. In Proceedings of the 14 th International Confer- ence on Informatics in Control (ICINCO), volume 2, pages 154-161. INSTICC, SciTePress.

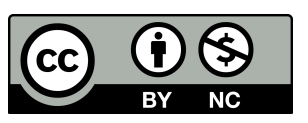

(C) 2018 by the authors. Submitted for possible open access publication under the terms and conditions of the Creative Commons Attribution $\mathrm{CC}-\mathrm{BY}-\mathrm{NC} \quad 3.0$ license (http://creativecommons.org/licenses/by-nc/3.0/). 